[CONTribution From the Chemical, Laboratory OF the Massachusetrs Institute OF TECHNOLOGY.]

\title{
THE CONDENSATION OF BENZOYL CHLORIDE WITH ETHYL- ENE IN THE PRESENCE OF ALUMINUM CHLORIDE.
}

By James F. Norris ANd Henry B. Couch.

Received July 19, 1920.

In an endeavor to correlate the chemical reactions of hydrocarbons and to determine the factors which influence the activity of hydrogen atoms in organic compounds, the conclusion was drawn that unsaturated radicals and aryl radicals have approximately the same activating influence on certain hydrogen atoms in compounds containing these groups. The ability of a hydrogen atom joined to a carbon atom to enter into a condensation of a definite type is dependent on the nature of the groups with which the carbon atom is linked. These groups differ in weight, negativity, unsaturation, and form as represented by the stereo-chemical effect and chain or ring structure; and they differ, no doubt, in other respects not understood at present. It is highly probable that what is commonly called negativity is a factor of the greatest importance. A study of the effect of the change in the negativity of the substituting radicals in compounds of a definite type on the activity of the carbonhydrogen bond has led to interesting results, and has suggested subjects for research which will throw additional light on the influence of structure on the chemical behavior of organic compounds.

The work described below was undertaken as a result of the fact that the vinyl and phenyl groups produce in compounds of different types the same general effect on the activity of certain atoms in these compounds. At present the best quantitative means of comparing the negativity of radicals is the ionization-constants of acids which contain these groups. The ionization-constant of benzoic acid is $6.6 \times 10^{-5}$ and that of acrylic acid is $5.6 \times 10^{-5}$. These figures indicate that when the hydrogen atom in formic acid is replaced by a phenyl or a vinyl radical acids are obtained which differ but slightly in activity as measured by their ionization in water. If such activity is traceable to negativity the 2 radicals differ but slightly in this quality. A comparison of the reactions of a number of compounds containing a phenyl radical and analogotss compounds containing a vinyl radical brought out the fact that the effect of these 2 groups was markedly similar. For example, vinyl bromide and phenyl bromide in many reactions behave alike. In both cases the halogen is relatively inactive towards reagents with which ethyl bromide and other saturated alkyl halides react. Again, the activity of the hydroxyl group in benzyl alcohol and that of the same group in allyl alcohol are strikingly alike in many respects. While methyl alcohol is not converted into methyl chloride in an open vessel by aqueous hydrochloric acid, benzyl alcohol, 
$\mathrm{C}_{6} \mathrm{H}_{5} \cdot \mathrm{CH}_{2} \mathrm{OH}$, and allyl alcohol, $\mathrm{CH}_{2}=\mathrm{CH} \cdot \mathrm{CH}_{2} \mathrm{OH}$, yield the chloride tnder these conditions. The activation produced by the 2 radicals, phexyl and vinyl, appear in this case to be similar.

From the above it appeared possible that a hydrogen atom in ethylene and one in benzene might show similar reactivity since in the first case the atom is linked to vinyl and in the second to phenyl. It seemed worth while to test this view. The most characteristic reaction of aromatic hydrocarbons is perhaps their ability to condense with acyl or alkyl halides in the presence of aluminum chloride. The Friedel-Crafts reaction has been applied almost exclusively to aromatic hydrocarbons and their derivatives. Accordingly, an attempt was made to condense benzoyl chloride with ethylene in the presence of aluminum chloride, Benzoyl chloride was chosen as it enters smoothly into condensations of the type to be studied and the compound expected, phenylvinyl ketone, had been previously prepared by other methods and its properties were known.

The condensation did take place and the product obtained had the properties of phenylvinyl ketone described by Tollens.

A search of the literature was made to determine if any reactions analogous to the above had been studied. A paper by Krapivin ${ }^{1}$ was found in which a similar condensation between acetyl halides and unsaturated hydrocarbons was described. The reactions studied were those between acetyl bromide and propylene and trimethylene, and between acetyl chloride and iso-butylene, trimethylene, hexylene, heptylene, and octylene. In all cases one of the hydrogen atoms of the hydrocarbons was replaced by the acyl radical.

It seems highly probable from these results that unsaturated compounds containing a double bond can replace aryl hydrocarbons in the FiriedelCrafts synthesis. This preliminary paper is published now as the authors cannot continue the work in common. The study of the reaction will be continued, as it opens up a new synthetic method which will prove of great value in the preparation of unsaturated compounds. It can also be applied to the synthesis of saturated hydrocarbons and their derivatives on account of the fact that the double bond can be readily reduced by the methods now available. It appears possible to make by this synthetic method compounds the preparation of which was difficult or impossible by the methods now in use. The behavior of alkyl halides, acyl halides, and polyhalogen derivatives will be studied with unsaturated hydrocarbons, including those containing aryl radicals.

Other reactions more or less characteristic of aromatic hydrocarbons will be studied to determine whether ethylene and its derivatives show a

Xrapivin, Bull, soc. imp, nat, Moscou, r, r76 (rgo8); Chem. Zentr, Igro, I, 335: C. A., $5,+285$, 
behavior analogous to such hydrocarbons. It is an interesting fact in this connection that phenyl ethylene yields a nitro compound of the structure $\mathrm{C}_{5} \mathrm{H}_{5} \mathrm{CH}_{2}=\mathrm{CHNO}_{2}$ when treated with nitric acid. In this case the nitration takes place in the side-chain. The work which is being done is part of an investigation which has as its aim the determination of the factors which influence the activity of the carbon-hydrogen bond in organic compounds.

\section{Experimental.}

The condensation of ethylene with benzoyl chloride was effected by first preparing the compound of the latter with aluminum chloride in the presence of carbon disulfide, and passing the gas at room temperature slowly through the mass of crystals covered with the liquid.

A mixture of $25 \mathrm{~g}$. of benzoyl chloride, $24 \mathrm{~g}$. of anhydrous aluminum chloride, and $600 \mathrm{cc}$. of carbon distlfide was heated to boiling for 2 hours in a flask provided with a reflux condenser. On cooling, a mass of nearly colorless crystals separated. Ethylene dried by bubbling through conc. sulfuric acid was next passed to the bottom of the flask containing the carbon disulfide and the crystals of the molecular compound. The gas was absorbed very slowly. When ro liters, which is about 2.5 molecules of ethylene to one of benzoyl chloride, had been passed through the mixture, the crystals had become covered with a dark, viscous liquid which interfered seriously with their coming into contact with the gas. The flask was then opened and the crystals crushed with a flattened glass rod. Ethylene was again passed through the mixture. Finally the reaction was stopped and the mixture poured onto ice. When the intermediate connound had decomposed the carbon disulfide was separated, poured through a dry filter-paper, and distilled. The residue was distilled with steam when an oil of characteristic sharp odor passed over along with some benzoic acid. On cooling the contents of the flask a large amount of benzoic acid crystallized out. This fact indicated that the reaction had not been complete. The oil volatile with steam was shaken with a dilute solution of sodium hydroxide and taken up with ether. On the evaporation of the solvent a pungent oil was obtained.

Moureau ${ }^{1}$ describes a compound as phenylvinyl ketone which he obtained by condensing the chloride of acrylic acid with benzene in the presence of aluminum chloride. The compound is slightly soluble in water from which it crystallizes at $4^{\circ}$ to $5^{\circ}$ in needles which melt at $42^{\circ}$, and forms a phenylhydrazone which melts at $130^{\circ}$.

Later Van Marle and Tollens ${ }^{2}$ and Schafer and Tollens ${ }^{3}$ describe as phenylvinyl ketone a compound which possessed properties different

1 Moreau, Ann. chim. phys., [7] 2, 199 (1894).

2 Van Marle and Tollens, Ber., 36, 1355 (1903).

\$Schafer and Tollens, ibid., 39, 2187 (1906). 
from those of the conpound isolated by Moureau. According to Tollens the ketone is a yellow oil, which could not be obtained as crystals. It had a characteristic, pungent odor and polymerized repidly to a very viscous resin. The phenyl-hydrazone prepared from the oil was obtained in well-defined yellow crystals, which melted at $152-153^{\circ}$ and gave a greenish blue fluorescent solution in alcohol.

The compound prepared by us possessed all the properties described by Tollens. The phenylhydrazone was prepared as follows. About I. $5 \mathrm{cc}$, of the oil was dissolved in $7 \mathrm{cc}$. of alcohol and treated with $2 \mathrm{cc}$. of freshly distilled phenylhydrazine. The mixture was heated on the steam-bath for half an hour. The crystals which separated were recrystallized from boiling alcohol. The compound, which was obtained in long, fine needies of a canary-yellow color, melted at $\mathrm{ISI}^{\circ}$ (uncorr.). The alcoholic solution of the phenylhydrazone showed a beautiful greenish bitue fluorescence at the surface of the liquid.

A solution of the oil in carbon tetrachloride decolorized bromine rapidly; in a short time hydrobromic acid was evolved. The oil slowly changed to a wax-like mass; this occurred more rapidly when it was allowed to stand overnight in the presence of water.

The yield of the ketone obtained was about $25 \%$; but it is highly probable that this could be markedly increased under better experimental conditions. There did not appear to be side reactions taking place in appreciable amounts, although a small quantity of a product insoluble in carbon disulfide was obtained. The main difficulties to overcome are to increase the rate of the absorption of the gas and to prevent the enclosure of the crystals of the addition-product of benzoyl chloride and aluminum chioride by the viscous reaction-product. The condensation will be studied more fully.

\section{Summary.}

On account of the similarity in the chemical behavior of elements joined to the vinyl and to the phenyl radicals an attempt was made to condense ethylene with benzoyl chloride by means of aluminum chloride. Phenylvinyl ketone was prepared in the same way. 